\title{
Successful Implementation of a Window for Routine Antimicrobial Prophylaxis Shorter than That of the World Health Organization Standard
}

\author{
Heidi Misteli, MD; ${ }^{1}$ Andreas F. Widmer, MD, MS; ${ }^{2}$ Walter P. Weber, MD; ${ }^{1}$ Evelyne Bucher, MD; ${ }^{3}$ \\ Marc Dangel, MPH, RN; ${ }^{2}$ Stefan Reck, MD; ${ }^{1}$ Daniel Oertli, MD, FACS; ${ }^{1}$ \\ Walter R. Marti, MD, FACS; ${ }^{4}$ Rachel Rosenthal, MD ${ }^{1}$
}

\begin{abstract}
OBJECTIVE. To evaluate the feasibility of implementation of the refined window for routine antimicrobial prophylaxis (RAP) of 30-74 minutes before skin incision compared to the World Health Organization (WHO) standard of 0-60 minutes.

DESIGN. Prospective study on timing of routine antimicrobial prophylaxis in 2 different time periods.

SETTING. Tertiary referral university hospital with 30,000 surgical procedures per year.

METHODS. In all consecutive vascular, visceral, and trauma procedures, the timing was prospectively recorded during a first time period of 2 years ( $A$; baseline) and a second period of 1 year ( $B$; after intervention). An intensive intervention program was initiated after baseline. The primary outcome parameter was timing; the secondary outcome parameter was surgical site infection (SSI) rate in the subgroup of patients undergoing cholecystectomy/colon resection.
\end{abstract}

RESULTS. During baseline time period A (3,836 procedures), RAP was administered 30-74 minutes before skin incision in 1,750 (41.0\%) procedures; during time period B ( 1,537 procedures), it was administered in $914(56.0 \% ; P<.001)$. The subgroup analysis did not reveal a significant difference in SSI rate.

CONCLUSIONS. This bundle of interventions resulted in a statistically significant improvement of timing of RAP even at a shortened window compared to the WHO standard.

Infect Control Hosp Epidemiol 2012;33(9):912-916

Surgical site infections (SSIs) significantly impact morbidity and mortality and represent a leading cause of healthcareattained infection..$^{1-3}$ Appropriate perioperative antimicrobial prophylaxis can reduce the incidence of SSI. ${ }^{4-6}$ Today, singleshot administration of a first- or second-generation cephalosporin is the state-of-the-art procedure in routine antimicrobial prophylaxis (RAP), ${ }^{7}$ supplemented with metronidazole in colorectal surgery for anaerobic coverage. In addition to the type of antibiotic, the timing of RAP in relation to skin incision is crucial. Based on the findings of the landmark publication in 1992 by Classen et al, ${ }^{8}$ guidelines recommend the administration of RAP within 2 hours before skin incision, whereas the 2008 World Health Organization (WHO) guidelines for safe surgery support administration within 1 hour before skin incision. ${ }^{9}$ In a prospective cohort study of 6,540 interventions, administration of RAP 30-74 minutes before skin incision achieved the lowest SSI risk. ${ }^{10}$
Therefore, local guidelines were released in 2009 at Basel University Hospital recommending RAP within 30-74 minutes.

This study was conducted in a cohort of patients who underwent operations after implementation of these guidelines to assess whether they resulted in an improvement of RAP timing (primary outcome). Additionally, the study aimed at comparing the 2 time periods regarding the SSI rates in the subgroup of patients undergoing cholecystectomy and colon resection (secondary outcome).

\section{METHODS}

\section{Patients and Procedures}

During time period A (January 1, 2000, to December 31, 2001) and time period B (April 1, 2009, to March 31, 2010), all consecutive surgical interventions performed in the vis-

Affiliations: 1. Division of General Surgery, University Hospital of Basel, Basel, Switzerland; 2. Division of Infectious Disease and Hospital Epidemiology, University Hospital of Basel, Basel, Switzerland; 3. Division of Anesthesiology, University Hospital of Basel, Basel, Switzerland; 4. Division of General Surgery, Kantonsspital Aarau, Aarau, Switzerland.

Received January 17, 2012; accepted April 5, 2012; electronically published July 24, 2012.

(C) 2012 by The Society for Healthcare Epidemiology of America. All rights reserved. 0899-823X/2012/3309-0008\$15.00. DOI: 10.1086/667374 
ceral, vascular, and trauma divisions at Basel University Hospital were prospectively recorded. Operations involving no incision (closed reductions of joint dislocations), hospital stay of less than 24 hours, and procedures classified as dirtyinfected were excluded. Administration of RAP was based on Centers for Disease Control and Prevention (CDC) guidelines for surgical wound classification ${ }^{11}$ and was administered in class I (clean) wounds involving an implant (eg, hernia mesh repair, trauma surgery), class II (clean-contaminated) procedures (eg, colorectal, biliary surgery), and class III (contaminated) procedures when the source of infection was surgically completely removed with no further need for antibiotic treatment. It consisted of single-shot cefuroxime in the case of colorectal surgery with coadministration of $500 \mathrm{mg}$ metronidazole and was repeated in the case of surgery exceeding 4 hours. For time period A, baseline data were retrieved from the prospective observational study. ${ }^{10}$ The interval between administration of RAP and incision was compared between cohort A and cohort $\mathrm{B}$. There was no change in practice of RAP over the investigated time periods.

The SSI rate in the subgroup of all patients undergoing laparoscopic or open cholecystectomy and colon surgery was compared between the 2 periods. Cholecystectomy was routinely performed laparoscopically with the exception of contraindications for pneumoperitoneum, intraoperative complications not easily manageable laparoscopically, or multiple previous surgeries with difficulties in establishing laparoscopic access, for which an open access/conversion was undertaken. As for colon surgery, the majority was carried out by open surgery. SSI was prospectively assessed by an independent team of infection control and prevention specialists and defined according to the guidelines of the CDC. ${ }^{11}$ The assessment was undertaken prospectively clinically for inpatients and for outpatient follow-up by telephone (up to 5 phone calls for contacting the patient and his or her family doctor, respectively) up to 30 days postoperatively.

The study was approved by the human subject committee and was part of the continuous quality improvement program, which was supported by the hospital executive board, as previously described. ${ }^{10}$ As an observational study within the quality improvement project, it was exempted from the written informed consent requirement.

\section{The 3 Interventions}

1. A consensus among all involved divisions was provided in oral and written form to all involved clinicians and published on the hospital intranet.

2. When applying for an operation room (OR) slot, the surgeons routinely had to indicate details on RAP, allowing the anesthesiologists to administer RAP prior to the surgeon being present in the $\mathrm{OR}$ and thus avoiding administration during the last 30 minutes before skin incision. The information was mandatory; otherwise, the application could not be processed. Before the intervention, surgeons were able to provide this information in the OR, frequently not allowing sufficient time for optimal RAP.

3. Anesthesiologists switched from a written chart to an electronic chart to record the time of RAP administration. Anesthesiologists were supported by the computer software that automatically reminded them to administer a second dose in cases of surgery exceeding 4 hours.

\section{Definitions}

The correct timing was defined (a) according to the Classen guidelines within 2 hours of prior incision, ${ }^{8}(b)$ according to the WHO guidelines within 1 hour, ${ }^{9}$ and $(c)$ according to the local guidelines within 30-74 minutes. ${ }^{10}$ The comparison of SSI was performed $(a)$ for all cholecystectomies/colon resections with the exception of class IV wounds and $(b)$ for class I and II wounds only, since antibiotic prophylaxis for class III wounds may be regarded as preemptive therapy.

\section{Statistical Analysis}

Statistical analyses were performed using the $t$ test or $\chi^{2}$ test as appropriate. Two-tailed tests were used. A $P$ value less than .05 was considered statistically significant. Statistical analyses were conducted with SPSS, version 19.0.

\section{RESULTS}

\section{Baseline Characteristics}

During time periods A and $\mathrm{B}$, a total of 10,095 consecutive invasive procedures (time period A, 6,540; time period B, $3,555)$ were performed; 5,898/10,095 (58.4\%) received RAP, 4,265 during time period $\mathrm{A}$ and 1,633 during time period $\mathrm{B}$. Of these, $67(1.6 \%)$ and $87(5.3 \%)$ procedures, respectively, received antibiotics more than 120 minutes before incision, $3,836(89.9 \%)$ and $1,537(94.1 \%)$ within 120 minutes, and $362(8.5 \%)$ and $9(0.6 \%)$ after incision.

Data on baseline characteristics are listed in Table 1 . Whereas the American Society of Anesthesiologists classification did not differ between the 2 periods, there was a small but significant decrease in mean age and an increase in body mass index (BMI). In addition, there was a relative increase of vascular procedures and a decrease of trauma procedures, a higher number of clean wounds, and a lower number of emergency procedures over time. In period A, 417 (10.9\%) patients had a BMI greater than 30 as compared to 284 $(18.5 \%)$ in period $B$.

\section{Comparison of Timing of RAP between the 2 Periods}

The impact of the intervention program was significant; the relative increase in compliance to this refined window of application was $36 \%$. A significant increase in timing within 120 minutes before incision was found, as well as a trend toward more frequent administration within 60 minutes before incision (Table 2). Overall, the RAP was administered at a median of 30.0 minutes (interquartile range [IQR], 
$15.0-45.0)$ before surgery and 34.3 minutes (IQR, 20.0-49.7) after the intervention. In elective procedures, RAP was administered at a median of 30.0 minutes (IQR, 15.0-45.0) in time period $A$ and improved to 39.1 (IQR, 27-52) in time period $B$, whereas in emergency procedures RAP was administered at a median of 25.0 (IQR, 15.0-40.0) and 24.2 (IQR, 15-38) minutes for period A and B, respectively. Regarding intraoperative redosing for procedures at least 4 hours in time period $\mathrm{A}(n=310 ; 8.1 \%)$, redosing was performed in $136(43.9 \%)$ procedures, whereas in time period B $(n=$ $58 ; 3.8 \%)$, redosing was performed in $22(37.9 \%)$.

\section{Emergency Procedures as Compared to Elective Procedures}

Emergency procedures were performed in 1,253 cases (29.4\%) during period A and 271 (16.6\%) during period B. The correct timing, as referred to 30-74 minutes, improved significantly over time in elective procedures from 1,277 (42.4\%) to $816(59.9 \%)$ procedures (relative risk reduction of $41 \%$; $P<.001$ ), whereas in emergency procedures, timing was optimal in time period A in $473(37.7 \%)$ procedures as compared to $98(36.2 \%)$ procedures in B (relative risk reduction of $-4 \%$ ), without statistical significance ( $P=.625$; Table 2$)$.

\section{SSI Rate after Cholecystectomy and Colon} Surgery (Subgroup Analysis)

After excluding wound class IV, a total of 483 interventions (242 cholecystectomies, 241 colon surgeries) were performed in period A. Out of these, 44 developed an SSI (9.1\%), 17 (7\%) after cholecystectomy and $27(11 \%)$ after colon surgery. During period B, 257 interventions (164 cholecystectomies, 93 colon surgery) were performed. Out of these, 25 developed an SSI (11.35\%), $9(5.5 \%)$ after cholecystectomy and 16 $(17.25 \%)$ after colon surgery.

There was no significant difference in SSI rates between the 2 time periods ( $P=.535$ for cholecystectomy, $P=$ .428 for colon surgery). When excluding wound classes III and IV, the overall SSI rate did not show any significant difference between the 2 time periods.

\section{DISCUSSION}

This study demonstrates an improvement of optimal timing of RAP administration according to international ( $0-2$ hours prior to incision $\left.{ }^{8,9}\right)$ and local (30-74 minutes ${ }^{10}$ ) guidelines after implementation of an intervention program to optimize timing of RAP in a university teaching hospital. In a subset of cholecystectomy and colon resection patients, there was no difference in SSI rate before versus after the implementation of these guidelines.

Since 1992, the international guidelines of correct timing of RAP were based on the landmark publication by Classen et $\mathrm{al}^{8}$ recommending timing within 2 hours before skin incision. In August 2008, the WHO guidelines ${ }^{9}$ recommending administration within 1 hour prior to skin incision were published as a report of the World Alliance for Patient Safety.

At Basel University Hospital, new guidelines were imple-

TABLE 1. Summary of Characteristics by Surgical Procedure of the 2 Study Populations at the 2 Different Time Intervals

\begin{tabular}{lccr}
\hline Characteristics & Time period A $(n=4,265)$ & Time period B $(n=1,633)$ & $P$ \\
\hline Mean age $( \pm$ SD), years & $59.1(19.2)$ & $57.3(18.6)$ & .001 \\
Female gender & $2,079(48.7)$ & $781(47.8)$ & .527 \\
Mean BMI \pm SD), kg/m ${ }^{2}$ & $25.0(4.8)$ & $26.2(5.2)$ & $<.001$ \\
Division of surgical specialty & & & \\
$\quad$ Visceral surgery & $1,643(38.5)$ & $609(37.3)$ & .384 \\
Traumatology & $1,936(45.4)$ & $617(37.8)$ & $<.001$ \\
Vascular surgery & $686(16.1)$ & $407(24.9)$ & $<.001$ \\
ASA score & & & \\
1 & $562(13.2)$ & $201(12.3)$ & .373 \\
2 & $2,006(47.0)$ & $766(46.9)$ & .930 \\
3 & $1,487(34.9)$ & $575(35.2)$ & .803 \\
4 & $201(4.7)$ & $72(4.4)$ & .619 \\
5 & $9(0.2)$ & $6(0.4)$ & .285 \\
Unknown & & $13(0.8)$ & \\
Wound classification & & & $<.001$ \\
I & $2,996(70.2)$ & $1,288(78.9)$ & $<.001$ \\
II & $762(17.9)$ & $210(12.9)$ & $<.001$ \\
III & $507(11.9)$ & $135(8.3)$ & $<.001$ \\
Emergency procedure & & & $<.001$ \\
Yes & $1,253(29.4)$ & $271(16.6)$ & $1,362(83.4)$ \\
No & $3,012(70.6)$ & & \\
\hline
\end{tabular}

NOTE. All data are no. (\%) unless otherwise indicated. ASA, American Society of Anesthesiologists; BMI, body mass index; SD, standard deviation. 
TA BLE 2. Summary of the Different Time Intervals of Prophylactic Antibiotic Administration of the 2 Study Populations for All Procedures, Elective and Emergency Procedures

\begin{tabular}{|c|c|c|c|}
\hline \multirow{2}{*}{ 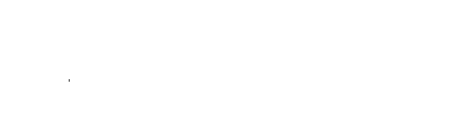 } & \multicolumn{3}{|c|}{$\begin{array}{c}\text { Guidelines (time interval), } \\
\text { minutes }\end{array}$} \\
\hline & $0-120$ & $0-60$ & $30-74$ \\
\hline \multicolumn{4}{|l|}{ All procedures } \\
\hline Time period A $(n=4,265), \%$ & 89.9 & 81.8 & 41.0 \\
\hline Time period $\mathrm{B}(n=1,633), \%$ & 94.1 & 83.6 & 56.0 \\
\hline Absolute RR, \% & 4.2 & 1.8 & 14.9 \\
\hline Relative RR, \% & 5 & 2 & 36 \\
\hline$P$ & $<.001$ & .099 & $<.001$ \\
\hline \multicolumn{4}{|l|}{ Elective procedures } \\
\hline Time period $\mathrm{A}(n=3,012), \%$ & 90.6 & 82.2 & 42.4 \\
\hline Time period B $(n=1,362), \%$ & 95.0 & 83.3 & 59.9 \\
\hline Absolute RR, \% & 4.4 & 1.2 & 17.5 \\
\hline Relative RR, \% & 5 & 1 & 41 \\
\hline$P$ & $<.001$ & .349 & $<.001$ \\
\hline \multicolumn{4}{|l|}{ Emergency procedures } \\
\hline Time period A $(n=1,235), \%$ & 88.4 & 80.8 & 37.7 \\
\hline Time period B $(n=271), \%$ & 89.7 & 84.9 & 36.2 \\
\hline Absolute RR, \% & 1.2 & 4.1 & -1.6 \\
\hline Relative RR, \% & 1 & 5 & -4 \\
\hline$P$ & .560 & .115 & .625 \\
\hline
\end{tabular}

NOTE. RR, risk reduction.

mented recommending an optimal window of 30-74 minutes before skin incision, referring to the published data by Weber et $\mathrm{al}^{10}$ in 2008. The results of this study show a statistically significant improvement of optimal timing overall and for elective surgery within this time frame after implementation of the new guidelines. Covering a time period of 12 months, this is likely to be a change in routine clinical practice rather than a short-term reaction to newly introduced guidelines. ${ }^{12,13}$ Although this improvement from $41 \%$ to $56 \%$ is statistically significant, there is an urgent need for additional strategies to improve administration within this time window and for validation of better outcomes in terms of SSI rates for the respective time window in a larger sample. We therefore plan to conduct a large randomized controlled trial to evaluate the optimal timing of RAP in terms of SSI as primary outcome. This study will be adequately constructed to deliver high-level evidence on the optimal timing.

The fact that no improvement was found for emergency surgery may be explained by the nature of emergency procedures, for which priority is given to immediately lifesaving interventions. Nevertheless, optimal timing remains important in emergency procedures as well, and improvement will require good interdisciplinary communication and standardized guidelines accounting for priorities in the emergency situation.

Concerning redosing, it is known that redosing of antibiotic prophylaxis in operations of at least 4 hours decreases the SSI risk. ${ }^{14}$ The compliance to these recommendations was low in both time periods. Although the rate is comparable with other published data, ${ }^{15}$ measures should be implemented to improve adequate redosing. An electronic reminder, which was installed during time period B in our operating theater, was helpful but frequently noticed only at the end of surgery. Combining it with an acoustic reminder would be a possibility to improve redosing.

The SSI rate after cholecystectomy and colon surgery was similar to the SSI rate found in literature, with up to $5.68 \%$ for cholecystectomy and $11.52 \%$ for colorectal surgery. ${ }^{16}$ Conversely, Smith et $\mathrm{al}^{17}$ found a rate of incisional SSI after elective colorectal resections of $26.5 \%$. Despite the significant improvement of RAP within the refined window, the SSI rate did not decrease between time period $\mathrm{A}$ and time period $\mathrm{B}$. A similar result was observed by Hawn et al. ${ }^{18}$ We hypothesize that the additional benefit of improved prophylaxis requires a larger sample size to detect small but clinically important differences, especially in patients undergoing elective cholecystectomy with a fairly low risk for SSI. This is also supported by the fact that there was only a small difference of 4.4 minutes in median time before incision between the 2 time periods. Additionally, prophylaxis was highly standardized and was not adapted to high BMIs. Moreover, other risk factors such as Staphylococcus aureus carriage ${ }^{19}$ and the lack of checklists ${ }^{20}$ possibly diminished the effects of RAP. Additionally, point prevalence studies performed routinely once or twice yearly by the infection control department clearly show a general trend for a continuous increase of nosocomial infections, likely representing the selection of seriously ill patients referred to the university hospital. Therefore, a stable incidence of SSIs may be considered a considerable success in an infection control program.

This study has several limitations. First, the prospective documentation of timing of RAP was manual in period A and electronic in period B. However, we do not assume an impact on the timing or its documentation, as previously described in a retrospective cohort study. ${ }^{21}$ Second, the SSI rate was studied in only a subset of patients. The subset of patients with cholecystectomy and colon resection was chosen as a sample for quality control in clean and clean-contaminated wounds. However, the SSI rate might have differed if other interventions had been accounted for.

In conclusion, our study showed the improvement of timing of RAP within 30-74 minutes prior to skin incision after implementation of new guidelines without change in SSI rates after cholecystectomy and colon resection. After further improvement of timing, a randomized-controlled trial will provide high-level evidence about optimal timing.

\section{ACKNOWLEDGMENTS}

This prospective cohort study was conducted as part of a quality improvement program at Basel University Hospital between 2000 and 2010, supported by the hospital executive board.

Financial support. This research was funded by the Department of Gen- 
eral Surgery, University Hospital of Basel, and by a grant of the Freiwillige Akademische Gesellschaft Basel. The grant funder had no role in design and conduct of the study; collection, management, analysis, and interpretation of the data; preparation, review, or approval of the manuscript.

Potential conflicts of interest. All authors report no conflicts of interest relevant to this article. All authors submitted the ICMJE Form for Disclosure of Potential Conflicts of Interest, and the conflicts that the editors consider relevant to this article are disclosed here.

Address correspondence to Heidi Misteli, MD, Department of General Surgery, University Hospital of Basel, Spitalstrasse 21, CH-4031 Basel, Switzerland (mistelih@uhbs.ch).

\section{REFERENCES}

1. Burke JP. Infection control: a problem for patient safety. $N$ Engl J Med 2003;348:651-656.

2. Kirkland $\mathrm{KB}$, Briggs JP, Trivette SL, et al. The impact of surgicalsite infections in the 1990s: attributable mortality, excess length of hospitalization, and extra costs. Infect Control Hosp Epidemiol 1999;20:725-730.

3. Gaynes RP, Culver DH, Horan TC, et al. Surgical site infection (SSI) rates in the United States, 1992-1998: the National Nosocomial Infections Surveillance System basic SSI risk index. Clin Infect Dis 2001;33(suppl 2):S69-S77.

4. Dellinger EP, Gross PA, Barrett TL, et al. Quality standard for antimicrobial prophylaxis in surgical procedures-the Infectious Diseases Society of America. Infect Control Hosp Epidemiol 1994; 15:182-188.

5. Kaiser AB. Antimicrobial prophylaxis in surgery. $N$ Engl J Med 1986;315:1129-1138.

6. Page CP, Bohnen JM, Fletcher JR, et al. Antimicrobial prophylaxis for surgical wounds: guidelines for clinical care. Arch Surg 1993;128:79-88.

7. Bratzler DW, Houck PM. Antimicrobial prophylaxis for surgery: an advisory statement from the National Surgical Infection Prevention Project. Clin Infect Dis 2004;38:1706-1715.

8. Classen DC, Evans RS, Pestotnik SL, et al. The timing of prophylactic administration of antibiotics and the risk of surgicalwound infection. $N$ Engl J Med 1992;326:281-286.

9. World Health Organization (WHO). WHO Guidelines for Safe Surgery, 2008. http://www.gawande.com/documents /WHOGuidelinesforSafeSurgery.pdf. Accessed May 23, 2011.

10. Weber WP, Marti WR, Zwahlen M, et al. The timing of surgical antimicrobial prophylaxis. Ann Surg 2008;247:918-926.

11. Mangram AJ, Horan TC, Pearson ML, et al. Guideline for prevention of surgical site infection, 1999-Centers for Disease Control and Prevention (CDC) Hospital Infection Control Practices Advisory Committee. Am I Infect Control 1999;27:97-132.

12. Pronovost $\mathrm{P}$, Needham D, Berenholtz $\mathrm{S}$, et al. An intervention to decrease catheter-related bloodstream infections in the ICU. $N$ Engl J Med 2006;355:2725-2732.

13. Pronovost PJ, Goeschel CA, Colantuoni E, et al. Sustaining reductions in catheter related bloodstream infections in Michigan intensive care units: observational study. BMJ 2010;340:c309.

14. Miliani K, L'heriteau F, Astagneau P. Non-compliance with recommendations for the practice of antibiotic prophylaxis and risk of surgical site infection: results of a multilevel analysis from the INCISO Surveillance Network. I Antimicrob Chemother 2009; 64:1307-1315.

15. Steinberg JP, Braun BI, Hellinger WC, et al. Timing of antimicrobial prophylaxis and the risk of surgical site infections: results from the trial to reduce antimicrobial prophylaxis errors. Ann Surg 2009;250:10-16.

16. National Nosocomial Infections Surveillance (NNIS) system report, data summary from January 1992 through June 2004, issued October 2004. Am J Infect Control 2004;32:470-485.

17. Smith RL, Bohl JK, McElearney ST, et al. Wound infection after elective colorectal resection. Ann Surg 2004;239:599-605.

18. Hawn MT, Itani KM, Gray $\mathrm{SH}$, et al. Association of timely administration of prophylactic antibiotics for major surgical procedures and surgical site infection. J Am Coll Surg 2008;206: 814-819.

19. Bode LG, Kluytmans JA, Wertheim HF, et al. Preventing surgicalsite infections in nasal carriers of Staphylococcus aureus. N Engl $J$ Med 2010;362:9-17.

20. de Vries EN, Prins HA, Crolla RM, et al. Effect of a comprehensive surgical safety system on patient outcomes. $N$ Engl J Med 2010;363:1928-1937.

21. de Vries EN, Dijkstra L, Smorenburg SM, et al. The Surgical Patient Safety System (SURPASS) checklist optimizes timing of antibiotic prophylaxis. Patient Saf Surg 2010;4:6. 